\title{
Activation of human natural killer cells by recombinant membrane-expressed fractalkine on the surface of tumor cells
}

\author{
XIAOJUN ZHANG, HAIMING WEI, QINGFENG CHEN and ZHIGANG TIAN
}

\begin{abstract}
Hefei National Laboratory for Physical Sciences at Microscale and School of Life Sciences, University of Science and Technology of China, Hefei 230027, P.R. China
\end{abstract}

Received December 4, 2006; Accepted January 30, 2007

\begin{abstract}
Chemokine receptors are typically expressed on natural killer cells, which can be activated by membrane ligands including the membrane chemokine fractalkine $(\mathrm{mFKN})$. This study investigated the function of mFKN on natural killer (NK) cell activation for interferon (IFN)- $\gamma$ production and cytotoxicity against tumors. HeLa cells were transfected with a membrane human fractalkine (mhFKN)expressing vector, and the transcription and surface expression of mhFKN in transfected HeLa cells were confirmed by RTPCR analysis and immunofluorescence assay, respectively. After co-culture of NK-92 cells with FKN-HeLa cells, the intracellular IFN- $\gamma$ in the NK-92 cells significantly increased compared to mock-HeLa cells. The concentration of IFN- $\gamma$ also increased in the supernatant of the NK-92 cells stimulated with FKN-HeLa cells. Moreover, the cytolytic activity of NK92 cells against K562 target tumor cells was significantly enhanced at each effector:target ratio in $4-\mathrm{h}{ }^{51} \mathrm{Cr}$-release assays when the NK-92 cells were pretreated with FKN$\mathrm{HeLa}$, indicating that membrane fractalkine activates the NK cells in the killing process. This study further confirms that membrane-expressed fractalkine plays a critical role in NK cell activation.
\end{abstract}

\section{Introduction}

Natural killer (NK) cells are thought to mediate immune responses against viral infection and neoplastic transformation by cytolytic activity without prior antigenic stimulation (1-8). NK cells are also believed to contribute to immunological regulation through the production of pro-

Correspondence to: Dr Zhigang Tian, School of Life Sciences, University of Science and Technology of China, 443 Huangshan Road, Hefei City, Anhui 230027, P.R. China

E-mail:tzg@ustc.edu.cn

Abbreviations: PBMC, peripheral blood mononuclear cells; mhFKN, membrane human fractalkine; IFN- $\gamma$, interferon- $\gamma$; NK, natural killer

Key words: chemokine, fractalkine, CX3CR1, natural killer cells, tumor inflammatory cytokines such as IFN- $\gamma$ and TNF- $\alpha$, which activate various types of cells including NK cells (9-11). Fractalkine (also named FKN or CX3CL1) is the only member of the CX3C subfamily, and exists in two isoforms: a membrane-anchored form and a soluble form that results from proteolytic cleavage (12-14). Membrane-bound FKN $(\mathrm{mFKN})$ directly mediates the capture and firm adhesion of leukocytes expressing its receptor CX3CR1 $(15,16)$. The expression pattern of CX3CR 1 in human NK cells is associated mostly with their cytotoxic effects (17). We have previously reported (18) that FKN is expressed in a variety of human tumor cell lines, and $\sim 90 \%$ of peripheral blood NK cells and all NK-92 cells express CX3CR1. The interaction between CX3CR1 on NK cells and FKN on tumor cells is involved in the natural cytotoxicity of NK cells against tumors. Here, we further showed that recombinant-expressed membrane human FKN (mhFKN) on HeLa cells activates NK cells against tumors and leads to a high production of IFN- $\gamma$.

\section{Materials and methods}

Antibodies and reagents. The polyclonal antibody against human fractalkine (goat $\mathrm{IgG}$ ) used in the indirect immunofluorescence assay was purchased from R\&D Systems (AF365). The secondary antibody (FITC-conjugated swine anti-goat $\mathrm{IgG}$ ) was purchased from BD Biosciences. The mAbs against human IFN- $\gamma$ and CD56 were purchased from BD Biosciences. ELISA kits for human IFN- $\gamma$ were purchased from Jingmei Biotech. The kit for reverse transcription was from Invitrogen and the PCR kit from Promega.

Cell lines and cell culture. NK cell line (NK-92) was obtained from ATCC. K562, a chronic myelogenous leukemia cell line, has been kept in our laboratory for many years. HeLa, a cervix epithelial tumor cell line, was also available from our institute. NK-92 cells were maintained in $\alpha$-minimum essential medium ( $\alpha$-MEM, Gibco BRL) without ribonucleosides and deoxyribonucleosides with $2 \mathrm{mM}$ L-glutamine adjusted to contain $1.5 \mathrm{~g} / 1$ sodium bicarbonate and supplemented with $0.2 \mathrm{mM}$ inositol, $0.1 \mathrm{mM}$ 2-mercaptoethanol, $0.02 \mathrm{mM}$ folic acid, $100 \mathrm{U} / \mathrm{ml}$ recombinant human IL-2 (PeproTech), $12.5 \%$ horse serum and $12.5 \%$ heatinactivated fetal bovine serum (both from Hyclone), $100 \mathrm{U} /$ $\mathrm{ml}$ penicillin and $100 \mathrm{U} / \mathrm{ml}$ streptomycin. K562 cells were 
cultured in RPMI-1640 medium (Gibco BRL) supplemented with $10 \%$ heat-inactivated fetal bovine serum, $2 \mathrm{mM}$ L-glutamine, $100 \mathrm{U} / \mathrm{ml}$ penicillin and $100 \mathrm{U} / \mathrm{ml}$ streptomycin. HeLa cells were maintained in Dulbecco's modified Eagle's medium (Gibco BRL) containing 10\% heat-inactivated fetal calf serum, $100 \mathrm{U} / \mathrm{ml}$ penicillin and $100 \mathrm{U} / \mathrm{ml}$ streptomycin.

Construction of recombinant membrane fractalkineexpressing vector. Firstly, the cDNA was synthesized with total RNA extracted from K562. Secondly, the complete ORF of human fractalkine (hFKN) was amplified from this cDNA by PCR using BamHI-tailed forward primer 5'-GG ATCCCATGGCTCCGATATCTCTGT-3' and EcoRI-tailed reverse primer 5'-GAATTCAGAGGAGTTCACACGGGC AC-3'. Finally the 1.1-kb BamHI-EcoRI fractalkine fragment was subcloned into a cloning site of pcDNA3 vector as described previously (19). Plasmid with low endotoxin was prepared on a large scale.

Cell transfection. The HeLa cells were transfected with the mhFKN-expressing vector (pcDNA3-fractalkine) using Lipofectamine 2000 (Invitrogen) following the manufacturer's instructions. Transfectants were selected by adding $1000 \mu \mathrm{g} /$ $\mathrm{ml} \mathrm{G} 418$ (Invitrogen) and then maintained with $500 \mu \mathrm{g} / \mathrm{ml}$ G418. Parent HeLa cells were also transfected with a mock vector (empty plasmid pcDNA3) as control.

Reverse transcription-polymerase chain reaction. Briefly, total RNA was isolated from HeLa cells with Trizol reagent (Invitrogen). Two micrograms of RNA were reversely transcribed with the random primer and incubated with $200 \mathrm{U}$ M-MLV for $1 \mathrm{~h}$ at $37^{\circ} \mathrm{C}$. Single-strand cDNA was then subjected to PCR. After a 2-min incubation at $95^{\circ} \mathrm{C}$, samples were added to $2 \mathrm{U}$ polymerase per $100 \mu \mathrm{l}$ reaction medium. The mixtures were then subjected to a 30 cycle-PCR amplification program consisting of denaturing for $30 \mathrm{sec}$ at $94^{\circ} \mathrm{C}$, annealing for $30 \mathrm{sec}$ at a different temperature, extension for $1 \mathrm{~min}$ at $72^{\circ} \mathrm{C}$, with a 7 -min extension after the last cycle. PCR primers for detecting transcription of human fractalkine, and $B$-actin were synthesized by Shanghai Sangon, Shanghai, P.R. China. Sequences of these primers were: human fractalkine (351 bp), forward 5'-AGGAGAATGCTCCGT CTGAA-3' and reverse 5'-AGAAGAGGAGGCCAAGGA AG-3'; and human B-actin (309 bp), forward 5'-GACCTG ACTGACTACCTCATGAAGAT-3' and reverse 5'-GTCAC ACTTCATGATGGAGTTGAAGG-3'.

Immunofluorescence microscopy. HeLa cells were cultured in a chamber slide (Nalge) and washed three times with icecold PBS. To avoid permeabilization of cell membranes, slides were fixed in PBS containing 4\% paraformaldehyde before the indirect immunofluorescence staining. These cells were then stained with the primary pAb against human fractalkine $(15 \mu \mathrm{g} / \mathrm{ml})$ or the isotype $\mathrm{IgG}$ as negative control. Finally, the cells were stained with the FITC-conjugated secondary antibody (swine anti-goat $\mathrm{IgG}$ ) (1:50 dilution).

Cell to cell stimulation. To determine the effect of membranebound fractalkine, NK-92 cells $\left(5 \times 10^{5}\right)$ were co-cultured with mock-HeLa cells or HeLa cells transfected with mhFKN
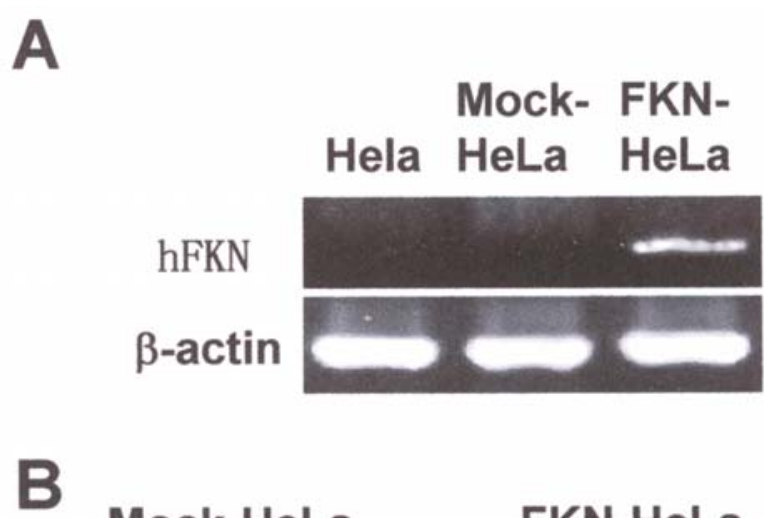

Mock-HeLa FKN-HeLa

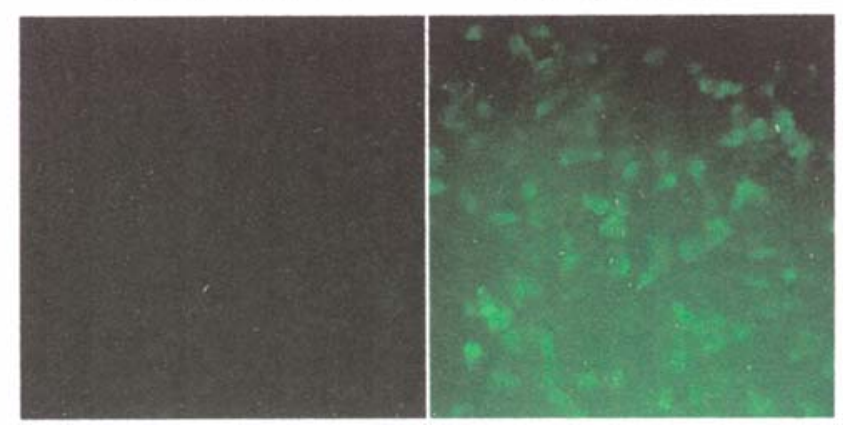

Figure 1. Recombinant surface expression of mhFKN on HeLa cells. (A) Total RNA was abstracted from HeLa cells transfected with pcDNA3-fractalkine or empty pcDNA3, then reversely transcripted (RT) into cDNA, and finally amplified by the PCR method. All RNA samples were pretreated with DNase before RT reaction to remove DNA contamination. (B) FKN-HeLa cells or mock-HeLa cells were stained with anti-human FKN pAb as described in Materials and methods. Fractalkine was detected on the surface of FKN-HeLa cells and not on the mock-HeLa cells.

$\left(5 \times 10^{5}\right)$ for $4 \mathrm{~h}$. To measure the intracellular cytokines of the NK-92 cells, monensin ( $2 \mu \mathrm{M}$, Sigma-Aldrich) and ionomycin (1 $\mu \mathrm{g} / \mathrm{ml}$, Sigma-Aldrich) were added to the medium, and the cells were cultured for an additional $4 \mathrm{~h}$. To measure the cytokines secreted by the NK-92 cells, the cells were cultured for another $4 \mathrm{~h}$ without any additions. After incubation, the cell deposits or cell-free supernatants were collected for further study.

Flow cytometric analysis. To examine the IFN- $\gamma$ produced in the NK-92 cells, the cells collected from the co-culture mixture described above were exposed to a standard staining protocol of intracellular cytokine detection (Current Protocols in Immunology). The cell mixture was washed 3 times with ice-cold PBS. Then the cells were fixed and permeabilized with a Cytofix/Cytoperm plus kit (BD Biosciences), and stained for intracellular IFN- $\gamma$. The mAb against human CD56 was added to identify the NK-92 cells in the mixture. The cells were analyzed by FACScalibur (BD Biosciences), and the data were analyzed using WinMDI 2.8 (free software for FACS analysis).

ELISA. To examine the IFN- $\gamma$ content in the supernatants of the NK-92 cell culture, the supernatants collected from the co-culture experiments mentioned above were assessed for IFN- $\gamma$ using ELISA kits according to the manufacturer's protocol. The detectable range of each ELISA kit was 5- 


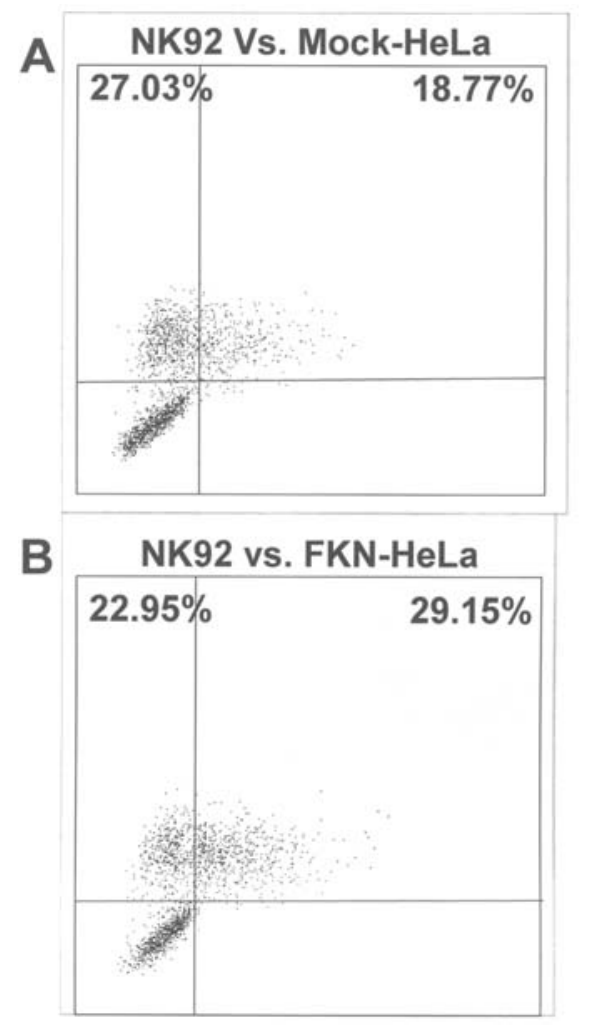

Figure 2. Enhancement of intracellular IFN- $\gamma$ production of NK-92 cells by stimulation of mhFKN on HeLa cells. NK-92 cells were incubated with confluent monolayers of mock-HeLa cells or fractalkine-transfected HeLa (FKN-HeLa) cells for $4 \mathrm{~h}$, and the secretion of IFN- $\gamma$ was blocked for another $4 \mathrm{~h}$ by monensin and ionomycin. Total IFN- $\gamma$ production of NK-92 cells was then analyzed with cytokine intracellular staining. (A) NK-92 cells were stimulated with mock-HeLa. (B) NK-92 cells were stimulated with FKNHeLa.

$1,000 \mathrm{pg} / \mathrm{ml}$, and the optical density of each individual well was determined at $450 \mathrm{~nm}$ using a microplate reader (BioTek Instrument Inc).

Cytotoxicity analysis. To determine the effect of mhFKN on NK-92 cell cytotoxicity, a modified $4-\mathrm{h}{ }^{51} \mathrm{Cr}$ release assay was performed. The HeLa cells during the logarithmic growth phase were collected, washed and plated into 96-well round-bottomed plates in varying numbers to achieve the desired 1:1 ratio with the NK-92 cells. These cells were incubated at $37^{\circ} \mathrm{C}$ in a humidified $5 \% \mathrm{CO}_{2}$ incubator for $5 \mathrm{~h}$ for recovery. Varying numbers of NK-92 cells were then added to the plates to achieve the desired E:T ratios, and these cells were co-cultured for $4 \mathrm{~h}$ for cell to cell stimulation. Finally, 1x10 ${ }^{41} \mathrm{Cr}$-labeled K562 cells [the K562 cells were collected and incubated in $100 \mu \mathrm{Ci}$ of $\mathrm{Na}^{51} \mathrm{Cr}$ (Perkin-Elmer Life Sciences) per $10^{6}$ cells, at $37^{\circ} \mathrm{C}$ in a humidified $5 \% \mathrm{CO}_{2}$ incubator for $1 \mathrm{~h}$ and washed three times with media] were added into each well. After a 4 -h incubation at $37^{\circ} \mathrm{C}$ in a humidified $5 \% \mathrm{CO}_{2}$ incubator, the supernatants were collected from each well and the radioactivity was determined using a $\gamma$-counter. Specific lysis $=[($ experimental $\mathrm{cpm}-$ spontaneous $\mathrm{cpm}) /($ maximum release $\mathrm{cpm}$ - spontaneous cpm)] x $100 \%$. To determine the effect of membrane fractalkine on NK-92 cell cytotoxicity against HeLa cells, a

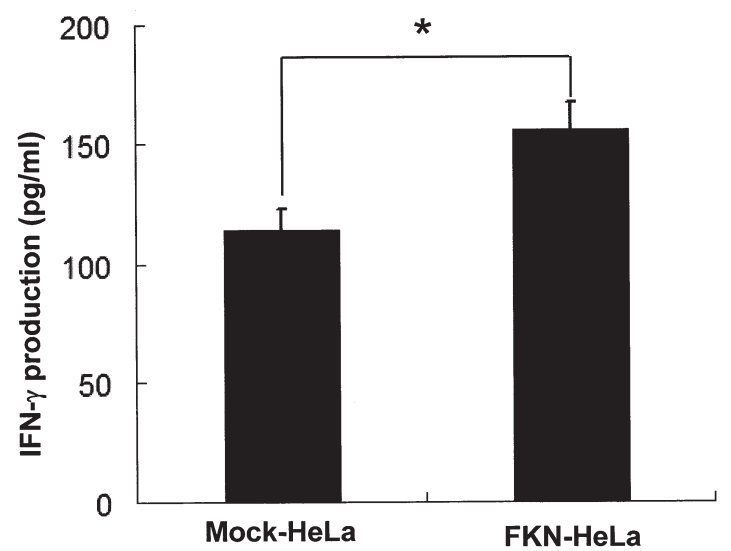

Figure 3. Enhancement of IFN- $\gamma$ secretion of NK-92 cells by stimulation of mhFKN on HeLa cells. NK-92 cells were incubated with confluent monolayers of control HeLa (mock-HeLa) cells or fractalkine-transfected HeLa (FKN-HeLa) cells for $8 \mathrm{~h}$ and secretion of IFN- $\gamma$ was then quantified with the ELISA method.

standard $4-\mathrm{h}{ }^{51} \mathrm{Cr}$ release assay was performed as described (20).

Statistical analysis. The Student's t-test for cytotoxicity was performed to determine statistical differences with $\mathrm{p}$ values $<0.05$ or $\mathrm{p}$ values $<0.01$ being considered significant or highly significant, respectively. All experiments were performed at least three times with a representative experiment being shown.

\section{Results}

Surface expression of human recombinant membrane fractalkine on HeLa cells. The complete ORF of the human fractalkine was cloned from K562 cells, which we previously reported to express membrane FKN (mhFKN) (18). The HeLa cells were transfected with a mhFKN-expressing vector, and the transcription of $\mathrm{mhFKN}$ in the transfected HeLa cells was examined by RT-PCR analysis. The PCR product with the predicted length was amplified from the total RNA of HeLa cells transfected with mhFKN (FKNHeLa), while no product was seen from those transfected with the mock vector (mock-HeLa) (Fig. 1A).

As reported, FKN exists as a membrane anchored form or as a soluble form. To determine whether the fractalkine in the transfected HeLa cells existed as the membrane-anchored form, we performed the immunofluorescence assay (Fig. 1B). Because the primary antibody reacts against the chemokine module that exists in both forms, we performed the assay following the protocol which was able to keep the integrity of the cellular membrane to ensure that the molecules we detected were those anchored to the membrane. As shown in Fig. 1B, recombinant mhFKN was detected on the surface of FKN-HeLa cells (mhFKN-HeLa), but not on the mock-HeLa cells.

Enhanced production of interferon- $\gamma$ by NK-92 cells after stimulation with recombinant membrane fractalkine. The activation of NK cells is critical in the early phase of immune 
A

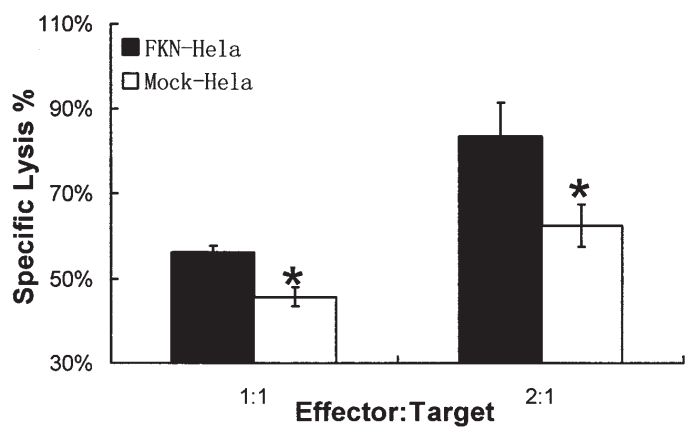

B

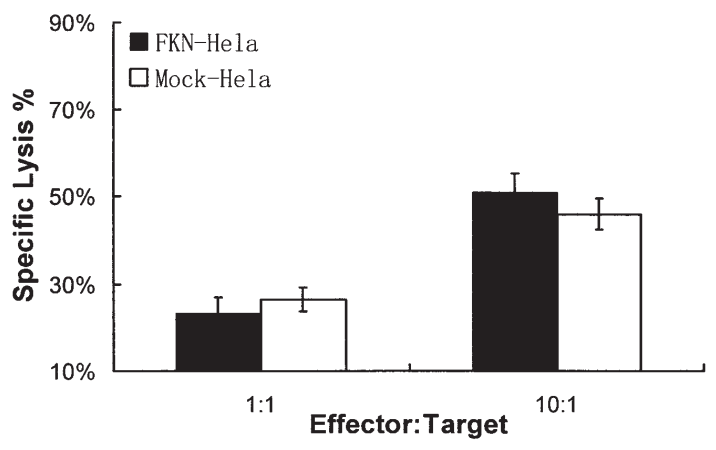

Figure 4. Improved cytotoxicity of NK-92 cells against K562 cells by stimulation of mhFKN on HeLa cells. The natural cytotoxic activity of NK92 cells against cancer cells was evaluated with the $4-\mathrm{h}{ }^{51} \mathrm{Cr}$-release assay at various effector/target ratios. (A) The natural cytotoxic activity of NK-92 cells against K562. NK-92 cells were pretreated with mock-HeLa cells or FKN-HeLa cells before the assay. (B) The natural cytotoxic activity of NK92 cells against mock-HeLa cells or FKN-HeLa cells. Values represent the means from at least 3 independent experiments.

responses against microbial infections. Production of IFN- $\gamma$ is an important event during NK activation $(4,21)$. Since NK92 cells express CX3CR1, the receptor for fractalkine as reported in our previous study, the membrane fractalkine of FKN-HeLa cells should activate NK-92 cells and enhance their production of IFN- $\gamma$. To explore this possibility, we performed co-culture experiments to measure the INF- $\gamma$ produced by NK-92 cells after stimulation with FKN-HeLa cells or mock-HeLa cells. As shown in Fig. 2, intracellular cytokine staining indicated that FKN-HeLa cells caused NK92 cells to synthesise more intracellular INF- $\gamma$ than mockHeLa cells did. Among the NK-92 cells stimulated by mockHeLa cells, $41 \%$ were IFN- $\gamma$ positive, while among the NK92 cells stimulated by mhFKN-HeLa cells, $56 \%$ were IFN- $\gamma$ positive. Meanwhile, we also examined the IFN- $\gamma$ content in the supernatant of the NK-92 cell culture (Fig. 3). The concentration of IFN- $\gamma$ was $156 \pm 12 \mathrm{pg} / \mathrm{ml}$ in the supernatant of NK-92 cells stimulated with mhFKN-HeLa cells; while that in the supernatant of mock-HeLa cells stimulated with NK-92 cells was $122 \pm 9 \mathrm{pg} / \mathrm{ml}$.

Improved cytotoxicity of NK-92 cells against tumor cells after stimulation with recombinant membrane fractalkine. Cytotoxicity is another feature of NK cells after activation. In order to directly determine the effect of membrane fractalkine on NK-92 cells, cytotoxicity of NK-92 cells was assayed in vitro. As shown in Fig. $4 \mathrm{~A}$, the natural cytotoxic activity of NK-92 cells pretreated with mhFKN-HeLa against K562 cells was significantly enhanced at each effector:target ratio in $4-\mathrm{h}{ }^{51} \mathrm{Cr}$-release assays, indicating that membrane fractalkine may activate the NK cells in the killing process. Notably, membrane fractalkine had no obvious effect on the cytotoxic activity against HeLa cells themselves (Fig. 4B), suggesting HeLa cells lacked other activating signals for mhFKNactivated NK-92 cells.

\section{Discussion}

In this study, we provided evidence that the FKN on tumor cells mediates antitumor activity by membrane interaction with human NK cells. This effect seems to rely solely on CX3CR1 on human NK cells. We previously reported in this journal (18) that FKN is expressed in a variety of human tumor cell lines, and $290 \%$ of peripheral blood NK cells and almost all NK-92 cells express CX3CR1. Anti-CX3CR1 antibody strongly inhibited the cytotoxicity of NK cells against K562 cells, and pretreatment of NK cells with recombinant soluble FKN enhanced the cytolytic function on tumor cells. Here, we further show that recombinant-expressed membrane human FKN on HeLa cells enhances NK cell cytotoxicity against K562 cells and causes high production of IFN- $\gamma$. It has also been reported that FKN immobilized by transfecting endothelial cells markedly induced IFN- $\gamma$ production by NK cells in a dose-dependent manner (22). In addition, transfection of FKN cDNA into ECV304 cells or HUVECs resulted in increased adhesion to NK cells and susceptibility to NK cell-mediated cytolysis (23). Moreover, both enhanced adhesion and susceptibility of fractalkinetransfected cells were markedly suppressed by anti-CX3CR1 antibody (23). These findings together demonstrate that surface FKN/CX3CR1 interaction plays an important role in the recruitment and adhesion of leukocytes including NK cells in inflammation.

Because several cytokines (IL-2, IFN- $\gamma$, and IL-12) and membrane ligands have been reported to enhance NK cytotoxicity $(24,25)$, we examined the functional roles of membrane fractalkine on NK cell activity. The mhFKN on HeLa cells significantly enhanced NK cell-mediated cytotoxicity against $\mathrm{K} 562$ target cells and IFN- $\gamma$ production from NK cells by both intracellular synthesis and secretion (Figs. 2 and 3). At present, the mechanisms of FKNmediated enhancement of NK cell activity are not clear. However, the functions of NK cells, such as cytotoxicity and cytokine production, are mediated by activation of several signaling molecules such as phospholipase $C_{\gamma}$, protein tyrosine kinases (PTKs), and phosphatidylinositol 3-kinase (PI3-K) (25-27). Pretreatment of NK cells with the phosphatidylinositol 3-kinase inhibitor completely inhibited the production of IFN- $\gamma$ induced by fractalkine (22).

As predicted by the missing self theory of immunosurveillance, initially proposed by Ljunggren and Karre (28), several families of NK inhibitory receptors which recognize allelic forms of MHC class I molecules may generate signals that inhibit NK cell-mediated cytotoxicity $(29,30)$. Therefore, the reason why the NK-92 cells activated by mhFKN-HeLa cells secreted more IFN- $\gamma$ without killing HeLa cells was because the HeLa cells kept high MHC class I molecules, unlike the K562 cells, though the NK-92 cells were totally activated (Fig. 4). The exact mechanisms underlying enhanced 
cytolysis against K562 cells after mhFKN recognition are not clear, but the FKN pathway activated in NK cells after mhFKN stimulation causing more production of IFN- $\gamma$ is an important finding.

\section{Acknowledgements}

This study was supported by the Natural Science Foundation of P.R. China (nos. 30528007, 30570819, 30571695, 30500467 and 30474585) and the Ministry of Education of P.R. China (no. 705029).

\section{References}

1. Trinchieri G: Biology of natural killer cells. Adv Immunol 47: 187-376, 1989

2. Unanue ER: Inter-relationship among macrophages, natural killer cells and neutrophils in early stages of Listeria resistance. Curr Opin Immunol 9: 35-43, 1997.

3. Scharton-Kersten TM and Sher A: Role of natural killer cells in innate resistance to protozoan infections. Curr Opin Immunol 9: 44-51, 1997.

4. Scott P and Trinchieri G: The role of natural killer cells in hostparasite interactions. Curr Opin Immunol 7: 34-40, 1995.

5. Hayakawa Y and Smyth MJ: NKG2D and cytotoxic effector function in tumor immune surveillance. Semin Immunol 18: 176-185, 2006.

6. Reyburn H, Mandelboim O, Vales-Gomez M, et al: Human NK cells: their ligands, receptors and functions. Immunol Rev 155: 119-125, 1997.

7. Costello RT, Fauriat C, Sivori S, Marcenaro E and Olive D: NK cells: innate immunity against hematological malignancies? Trends Immunol 25: 328-333, 2004.

8. Rajagopalan S and Long EO: Viral evasion of NK-cell activation. Trends Immunol 26: 403-405, 2005.

9. Kos FJ: Regulation of adaptive immunity by natural killer cells. Immunol Res 17: 303-312, 1998.

10. Le PC, Genin P, Baines MG and Hiscott J: Interferon activation and innate immunity. Rev Immunogenet 2: 374-386, 2000.

11. Zingoni A, Sornasse T, Cocks BG, Tanaka Y, Santoni A and Lanier LL: NK cell regulation of T cell-mediated responses. Mol Immunol 42: 451-454, 2005.

12. Tsou CL, Haskell CA and Charo IF: Tumor necrosis factoralpha-converting enzyme mediates the inducible cleavage of fractalkine. J Biol Chem 276: 44622-44626, 2001

13. Imai T, Hieshima K, Haskell C, et al: Identification and molecular characterization of fractalkine receptor CX3CR1, which mediates both leukocyte migration and adhesion. Cell 91: 521-530, 1997.

14. Garton KJ, Gough PJ, Blobel CP, et al: Tumor necrosis factoralpha-converting enzyme (ADAM17) mediates the cleavage and shedding of fractalkine (CX3CL1). J Biol Chem 276: 37993-38001, 2001.
15. Umehara $\mathrm{H}$ and Imai $\mathrm{T}$ : Role of fractalkine in leukocyte adhesion and migration and in vascular injury. Drug News Perspect 14: 460-464, 2001.

16. Fong AM, Robinson LA, Steeber DA, et al: Fractalkine and CX3CR 1 mediate a novel mechanism of leukocyte capture, firm adhesion, and activation under physiologic flow. J Exp Med 188: 1413-1419, 1998.

17. Xin H, Kikuchi T, Andarini S, et al: Antitumor immune response by CX3CL1 fractalkine gene transfer depends on both NK and T cells. Eur J Immunol 35: 1371-1380, 2005.

18. Zhang X, Wei H, Wang H and Tian Z: Involvement of interaction between Fractalkine and CX3CR1 in cytotoxicity of natural killer cells against tumor cells. Oncol Rep 15: 485-488, 2006.

19. Ben-Baruch A, Xu L, Young PR, Bengali K, Oppenheim JJ and Wang JM: Monocyte chemotactic protein-3 (MCP3) interacts with multiple leukocyte receptors. C-C CKR1, a receptor for macrophage inflammatory protein-1 alpha/Rantes, is also a functional receptor for MCP3. J Biol Chem 270: 22123-22128, 1995.

20. Sarin A, Williams MS, Alexander-Miller MA, Berzofsky JA, Zacharchuk CM and Henkart PA: Target cell lysis by CTL granule exocytosis is independent of ICE/Ced-3 family proteases. Immunity 6: 209-215, 1997

21. Seaman WE: Natural killer cells and natural killer T cells. Arthritis Rheum 43: 1204-1217, 2000.

22. Yoneda $\mathrm{O}$, Imai $\mathrm{T}$, Nishimura $\mathrm{M}$, et al: Membrane-bound form of fractalkine induces IFN-gamma production by NK cells. Eur J Immunol 33: 53-58, 2003.

23. Yoneda O, Imai T, Goda S, et al: Fractalkine-mediated endothelial cell injury by NK cells. J Immunol 164: 4055-4062, 2000.

24. Lin SJ, Roberts RL, Ank BJ, Nguyen QH, Thomas EK and Stiehm ER: Human immunodeficiency virus (HIV) type-1 GP120-specific cell-mediated cytotoxicity (CMC) and natural killer (NK) activity in HIV-infected (HIV+) subjects: enhancement with interleukin-2(IL-2), IL-12, and IL-15. Clin Immunol Immunopathol 82: 163-173, 1997.

25. Biron CA, Nguyen KB, Pien GC, Cousens LP and Salazar-Mather TP. Natural killer cells in antiviral defense: function and regulation by innate cytokines. Annu Rev Immunol 17: 189-220, 1999.

26. Leibson P: Viewpoint: signal transduction during natural killer cell activation. Nat Immun 14: 117-122, 1995.

27. Lanier LL: Natural killer cell receptor signaling. Curr Opin Immunol 15: 308-314, 2003

28. Ljunggren HG and Karre K: In search of the 'missing self': MHC molecules and NK cell recognition. Immunol Today 11: 237-244, 1990.

29. Moretta A, Bottino C, Vitale M, et al: Activating receptors and coreceptors involved in human natural killer cell-mediated cytolysis. Annu Rev Immunol 19: 197-223, 2001.

30. Raulet DH, Vance RE and McMahon CW: Regulation of the natural killer cell receptor repertoire. Annu Rev Immunol 19: 291-330, 2001 